\title{
Plasmodium falciparum $K 13$ expression associated with parasite clearance during artemisinin-based combination therapy
}

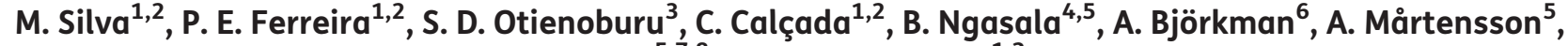 \\ J. P. Gil ${ }^{5,7,8}$ and M. I. Veiga (D) ${ }^{1,2 *}$ \\ ${ }^{1}$ Life and Health Sciences Research Institute (ICVS), School of Medicine, University of Minho, Campus de Gualtar, 4710-057, Braga, \\ Portugal; ${ }^{2} I C V S / 3 B$ 's - PT Government Associate Laboratory, Braga/Guimarães, Campus de Gualtar, 4710-057, Braga, Portugal; \\ ${ }^{3}$ Department of Computer Science and Engineering, Johnson C. Smith University, Charlotte, NC 28216, USA; ${ }^{4}$ Muhimbili University of \\ Health and Allied Sciences, Dar es Salaam, Tanzania; ${ }^{5}$ Department of Women's and Children's Health, International Maternal and \\ Child Health (IMCH), Uppsala University, Uppsala, Sweden; ${ }^{6}$ Malaria Research Unit, Department of Microbiology, Tumour and Cell \\ Biology, Karolinska Institutet, Stockholm, Sweden; 'Division of Pharmacogenetics, Department of Physiology and Pharmacology, \\ Karolinska Institutet, Stockholm, Sweden; ${ }^{8}$ Center for Biodiversity, Functional \& Integrative Genomics, Faculdade de Ciências, \\ Universidade de Lisboa, Lisbon, Portugal \\ *Corresponding author. Life and Health Sciences Research Institute (ICVS), School of Medicine, University of Minho, Campus de Gualtar, 4710-057, \\ Braga, Portugal. Tel: +351 253604 834; Fax: +351 253604 809; E-mail: mariaveiga@med.uminho.pt (D) orcid.org/0000-0002-2205-8102
}

Received 2 November 2018; returned 16 December 2018; revised 1 February 2019; accepted 18 February 2019

Background: Delayed parasite clearance and, consequently, reduced efficacy of artemisinin-based combination therapies have been linked with Plasmodium falciparum K13 gene SNPs in Southeast Asia. In Africa, significantly prolonged clearance has not yet been observed and the presently restricted variation in parasite clearance cannot be explained by K13 polymorphisms.

Objectives: Our aim was to study the in vivo pfK13 transcriptional response in patients treated with artemetherlumefantrine and explore whether the pfk13 transcripts can explain the patients' parasite clearance outcomes.

Patients and methods: A total of 47 Tanzanian children with microscopically confirmed uncomplicated $P$. falciparum malaria were hospitalized and received artemether-lumefantrine treatment (clinical trial ID: NCT00336375). RNA was extracted from venous blood samples collected before treatment initiation and at five more timepoints after treatment. CDNA was synthesized and pfk13 transcripts measured by real-time PCR.

Results: A wide range of $p f k 13$ transcript variation was observed throughout all timepoints after artemetherlumefantrine treatment. Taking parasite clearance data together with the $\mathrm{pfk} 13$ transcripts profile, we observed a negative correlation inferring that $p f k 13$ down-regulation is associated with longer parasite clearance time.

Conclusions: The findings suggest that a reduced PfK13 transcriptional response may represent a first step towards artemisinin tolerance/resistance.

\section{Introduction}

Sustained artemisinin combination therapy (ACT) efficacy is dependent on the actions of both the long half-life component and the artemisinin derivative.

In Southeast Asia, a change in the parasite response to artemisinins has been observed, essentially characterized by a significant increase in infection clearance times. ${ }^{1,2}$ This phenotype has also been associated with clinical failure after ACT treatment. ${ }^{1,3,4}$ Of particular concern is the possibility that the reduction of artemisinin efficacy may also spread to Africa, where $90 \%$ of all malaria deaths occur, jeopardizing the United Nations sustainable developmental goal of 'ending the malaria epidemic' by $2030 .{ }^{5}$
Increased parasite clearance (PC) times have been firmly associated with sequence variation in the gene coding for the cytoplasmic K13 propeller-like protein (PF3D7_1343700). ${ }^{6}$ Genetically modified $\mathrm{K} 13$ loci confirmed the role of K13 propeller mutations in artemisinin resistance. Intriguingly, it was also observed that the levels of artemisinin resistance exhibited by strains with genetically modified K13 were lower compared with those of clinical isolates carrying the same mutations, ${ }^{7}$ indicating the existence of other determinants beyond the known mutations. This is supported by field reports of artemisinin resistance independent of K13 mutations, emphasizing the need for an understanding of resistance factors beyond K13 mutations. ${ }^{8}$ One possibility barely studied is the influence of in vivo K13 expression levels, prior to treatment 
and after artemisinin impact. In this work, we have studied the in vivo pfK13 expression in Tanzanian patients treated with artemether-lumefantrine and explore its association with patients' PC parameter outcomes.

\section{Methods}

\section{Study site and sample collection}

The trial under study, described in detail elsewhere, was conducted at the Fukayosi Primary Health Care Centre, Bagamoyo District, Tanzania ${ }^{9}$ in accordance with the Declaration of Helsinki and Good Clinical Practice. It was granted ethics clearance by the National Institute for Medical Research, Dar es Salaam, Tanzania, and the Regional Ethics Committee, Stockholm, Sweden. Informed consent was obtained from parents/guardians of all children enrolled [Clinical Trials (US), identifier NCT00336375]. Briefly, 50 children with microscopically confirmed uncomplicated Plasmodium falciparum malaria were hospitalized and received artemether-lumefantrine treatment in six doses over 3 days. Venous blood samples were collected before treatment initiation $(0 \mathrm{~h})$ and at nine more timepoints $(2,4,8,16,24$, $36,48,60$ and $72 \mathrm{~h}$ ) after initiation of treatment. Parasite densities were determined by Giemsa staining, and PC was measured and documented as the proportion of patients with positive microscopy. ${ }^{9}$

\section{Molecular analysis}

RNA was extracted from venous blood of the 50 enrolled patients using an ABIPRISM H6100 Nucleic Acid PrepStation (Applied Biosystems, Fresno, CA, USA). Not expecting to find parasitic RNA $24 \mathrm{~h}$ post-treatment, total RNA (human and parasite) quality and quantity were measured using the AgilentRNA6000Pico total RNA assay in an Agilent 2100 Bioanalyser $^{\mathrm{TM}}$ (Agilent, Santa Clara, CA, USA) and standardized prior to cDNA synthesis for the first six timepoints [ $\mathrm{h}$ (before treatment) and 2, 4, 8, 16 and $24 \mathrm{~h}$ after treatment initiation]. Three out of 50 patients sampled had low total RNA quality and therefore were excluded from further transcript analysis.

Quantitative PCR was performed in triplicate with custom MGB TaqMan ${ }^{\circledR}$ probes for the K13 gene, in an ABIPRISM ${ }^{\circledR} 7900 \mathrm{HT}$ Sequence Detection System (Applied Biosystems). The housekeeping gene serine-tRNA ligase (PF3D7_0717700), shown to be transcribed stably throughout different intraerythrocytic stages, ${ }^{10-13}$ was used as an endogenous control (normalizer).

Oligonucleotides were as follows: K13 6-FAM, NFQ probe, 5'ACGCCAGCATTGTTG-3'; PF3D7_0717700 VIC, TAMRA probe, 5'-TGAAAC TATAGAATCAAAAAGGTTACCACTCAAATACGCT)-3'; K13 primers, fw 5'-GTG GATTTGATGGTGTAGAATATTTAAATTCGA-3', rev 5'-GCTTTTTTGGTAGACATAG GTGTACACA-3'; and PF3D7_0717700 primers, fw 5'-CCTCAGAACAACC ATTATGTGCTT-3', rev 5'-TGTGCCCCTGCTTCTTTTCTA-3'. Amplification conditions were 40 cycles of $95^{\circ} \mathrm{C}(15 \mathrm{~s})+60^{\circ} \mathrm{C}(1 \mathrm{~min})$. For each patient, $p f K 13$ transcript fold change in expression was calculated by the $2^{-\Delta \Delta C t}$ method $^{14}$ in which the threshold cycle number (Ct) was normalized to the $\mathrm{Ct}$ of the housekeeping gene (PF3D7_0717700) for all timepoints ( $\Delta \mathrm{Ct}$ ) and $\Delta \Delta \mathrm{Ct}$ calculated using the $\Delta \mathrm{Ct}$ from timepoint $\mathrm{O} \mathrm{h}$ (before treatment) as a calibrator. Spearman correlation was applied to assess linear relationships between pfk13 expression variation throughout time and between PC parameters. Patient data were stratified in two groups defined as pfK13 transcript expression of more or less than 1 -fold change (1-fold change represents no pfK13 transcript difference after treatment; $\Delta \Delta \mathrm{Ct}=0 ; 2^{\circ}=1$ ). The MannWhitney test was used on the patient and PC data grouped with more or less than 1-fold change of pfk13 transcripts. The Cox proportional hazards analysis (Kaplan-Meier curve) was used to assess the effect of pfk13 transcripts on the $\mathrm{PC}_{50 \%}$ outcome. The analyses were done with GraphPad Prism version 7.00 software.

Sequencing of the $p f k 13$ resistant loci, encompassing the SNP PfK13 C580Y, was performed in all infections using the primers $5^{\prime}$-CAA
ATATTGCTACTGAAACTATG-3' and 5'-TGTGCATGAAAATAAATATTAAAGAAG$3^{\prime}$, and sequencing primer 5'-AGGTGGATTTGATGGTGTAGAA-3'.

\section{Results}

We obtained pfk13 transcript expression data for the 47 infections analysed at the six timepoints under review, with the exception of the $24 \mathrm{~h}$ point, at which 3 of the 47 infections did not provide reliable $p f k 13$ transcript results.

After artemether-lumefantrine treatment initiation, a wide range of pfk13 transcript fold change in expression was observed throughout all timepoints (Figure 1a). Using the pfk13 expression levels of each infection before treatment initiation $(0 \mathrm{~h})$ to understand the fold change in expression after treatment initiation, the expression ranged from 0.2 - to 4.1 -fold after $2 \mathrm{~h}, 0.1$ - to 4.4 -fold after $4 \mathrm{~h}, 0.1$ - to 8.3 -fold after $8 \mathrm{~h}, 0.1$ - to 7.7 -fold after $16 \mathrm{~h}$ and 0.04- to 6.1-fold after $24 \mathrm{~h}$.

Strong correlations of $p f k 13$ expression were observed between all post-treatment timepoints (Figure 1b), with individual infection variation defined at the first timepoint after treatment ( $2 \mathrm{~h}$ ) and maintained over time (Figure 1a; black connection lines). The data were not normally distributed (Shapiro-Wilk, $W=0.47-0.66$, $P<0.0001$ ), showing skewness for decreased expression of $p f k 13$ response (Figure 1a; yellow bar showing median values). This observed differential expression was not linked to genetic variability at the $p f k 13$ gene-resistant loci since all infections were found to be WT from amino acid 464 up to the $3^{\prime}$-terminus of the ORF.

By taking PC data (described in Table 1 ) together with the pfk13 expression profile over time, we noticed a clear clade based on Spearman correlation inferring that $p f k 13$ down-regulation is associated with longer PC time (Figure $1 b$ ). To confirm this, infections were stratified into two groups defined by infections with increased or decreased expression of pfk13 by more than or less than 1-fold, respectively (Figure 1a, red dashed line cut-off of 1 signifying no variation compared with before treatment initiation; $2^{-\Delta \Delta \mathrm{Ct}}=2^{\circ}$ ). Reinforcing the negative correlation result (Figure $1 \mathrm{~b}$ ), after $2 \mathrm{~h}$ of treatment, a significant difference $(P<0.001)$ in $\mathrm{PC}$ times was observed, with higher mean $\mathrm{PC}_{50 \%}$ values in the infections group with reduced $p f k 13$ expression. This significant difference was maintained for the $\mathrm{PC}_{90 \%}, \mathrm{PC}_{95 \%}$ and $\mathrm{PC}_{99 \%}$, but did not reach significance for the PC slope half-life (Table 1 ). To better understand the risk factor of the two groups $(p f k 13<1$ and pfk13 > 1) to predict the PC time, we performed a Kaplan-Meier survival analysis using the $P C_{50 \%}$. Significant difference in survival time for $\mathrm{PC}_{50 \%}$ is observed between the two groups (median $p f k 13<1=7.1 \mathrm{~h} ; \mathrm{pfk13}>1=1.9 \mathrm{~h}$ ) (Figure 1c).

\section{Discussion}

SNPs at PfK13 have been identified as molecular markers of ACT resistance among Southeast Asian patients. ${ }^{15}$ These have been found in $<3 \%$ in Africa, ${ }^{16}$ possibly explaining the lack of extreme cases of long PC time. Variation in this phenotype nevertheless exists and has been well documented. ${ }^{17}$ The question remains of whether there are other factors beyond ORF variation that might explain clearance differences in Africa. In this molecular prospective approach, we explored for the first time the transcriptional profile of pfk13 during artemether-lumefantrine therapy. Since transcriptional variation appears to be higher than genetic 
(a)

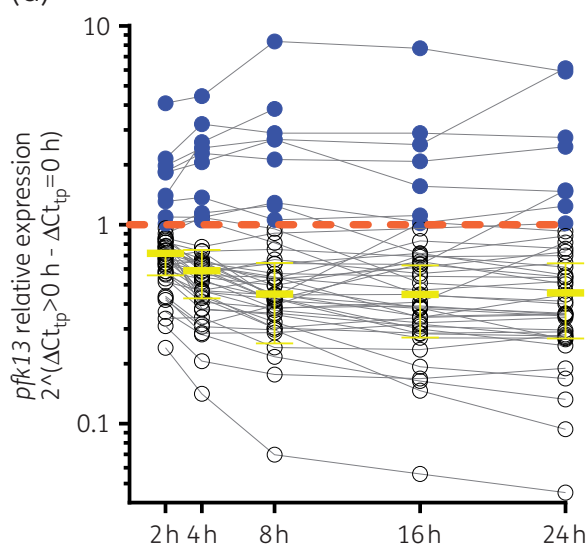

(b)

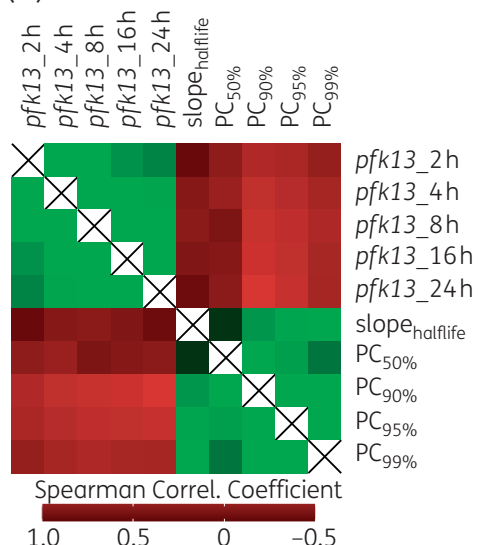

(c)

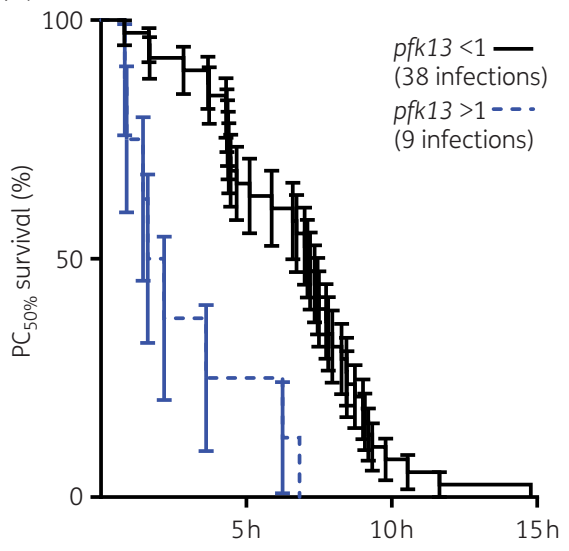

Figure 1. Changes in pfk13 transcripts and PC in patients treated with artemether-lumefantrine. (a) pfk13 relative expression by fold change after treatment compared with before treatment for every analysed patient sample over time. Connection lines demark individual patient samples over time. The dashed red line delineates the threshold for pfk13 up-regulation (blue circles, $>1$-fold) and down-regulation (open circles, $<1$-fold). Median pfk13 expression by timepoint is represented with a yellow dash, and standard error of the 47 samples. pfk13 expression fold change was calculated by the $2^{-\Delta \Delta \mathrm{Ct}}$ method normalized with the housekeeping gene PF3D7_0717700 and calibrated with paired infection data before treatment initiation (time 0 h). (b) The heat map shows the Spearman correlation coefficients of pairwise comparisons of the pfk13 relative expression at different timepoints and the PC parameters of matching patients. (c) Kaplan-Meier survival for $\mathrm{PC}_{50 \%}$ in the two groups defined in (a). $\chi^{2}$ [Log-rank (Mantel-Cox) test]: $17.7 ; P<0.0001 . P C_{50 \%}$ median survival: $p f k 13<1=7.1 \mathrm{~h} ; p f k 13>1=1.9 \mathrm{~h}$. This figure appears in colour in the online version of JAC and in black and white in the print version of JAC.

Table 1. Characteristics of the studied population and differential pfk13 expression

\begin{tabular}{|c|c|c|c|c|c|c|c|c|}
\hline & \multicolumn{3}{|c|}{ Total $(n=47)$} & \multicolumn{2}{|c|}{$p f k 13<1^{a}(n=38)$} & \multicolumn{2}{|c|}{$p f k 13>1^{a}(n=9)$} & \multirow[b]{2}{*}{$P$ value } \\
\hline & mean & SD & range & mean & SD & mean & SD & \\
\hline Age (months) & 50.0 & 30.0 & $12-119$ & 50.7 & 28.6 & 46.9 & 37.1 & 0.510 \\
\hline Weight (kg) & 14.3 & 5.5 & $8-30$ & 14.3 & 5.4 & 14.0 & 6.4 & 0.474 \\
\hline Parasite density (Pf/mm³ blood) & 60171 & 51805 & $2120-200400$ & 61744 & 50076 & 53529 & 61420 & 0.397 \\
\hline Temperature $\left({ }^{\circ} \mathrm{C}\right)$ & 37.9 & 1.0 & $36.2-40.8$ & 38.0 & 1.1 & 37.7 & 0.5 & 0.995 \\
\hline Slope half-life & 2.5 & 1.1 & $0.5-5.2$ & 2.6 & 1.1 & 2.1 & 1.1 & 0.343 \\
\hline$P C_{50 \%}(h)$ & 6.0 & 3.2 & $0.8-14.8$ & 6.7 & 2.9 & 3.0 & 2.4 & 0.0008 \\
\hline$P C_{90 \%}(h)$ & 11.6 & 4.8 & $2.1-21.8$ & 12.6 & 4.3 & 7.3 & 4.8 & 0.007 \\
\hline $\mathrm{PC}_{95 \%}(\mathrm{~h})$ & 14.1 & 5.7 & $2.6-26.2$ & 15.2 & 5.2 & 9.4 & 5.8 & 0.016 \\
\hline $\mathrm{PC}_{99 \%}(\mathrm{~h})$ & 19.9 & 8.0 & $3.9-37.8$ & 21.2 & 7.5 & 14.4 & 8.3 & 0.052 \\
\hline
\end{tabular}

${ }^{a} p f k 13$ expression profile after $2 \mathrm{~h}$ treatment. pfk13 $>1$ represents the infection group with fold change in relative expression above 1 , and pfk13<1 represents the infection group with fold change in relative expression below 1. Pf, P. falciparum.

variation in our study, we have hypothesized that it might have an important role in the early stage of development of drug resistance.

Previously, a large ex vivo transcriptomic study of P. falciparum isolates from Southeast Asian and African patients, collected before treatment and encompassing normal and delayed PC time, revealed high variation in PfK13 expression, although not correlated with artemisinin resistance. ${ }^{18}$ Our prospective study describing $p f k 13$ expression during treatment also showed a large range of transcription levels, with 20 times the dynamic range observed after $2 \mathrm{~h}$ of treatment. Interestingly, the intrinsic transcription features of each individual infection prevailed during the $24 \mathrm{~h}$ of study (Figure 1a).
The K13 propeller domain encodes six Kelch motifs theoretically playing a role in protein degradation via polyubiquitination or involved in cell response to oxidative stress. The C580Y mutation was shown to decrease affinity for a protein substrate, thereby increasing its steady-state levels by reducing ubiquitination and proteasomal degradation. ${ }^{19}$ Parasites responding with decreased expression of pfk13 could therefore act as a similar outcome, translated into a slow phenotype and manifested as longer PC times. This hypothesis could explain the clear negative correlation observed between the clearance parameters of the infections herein analysed and their pfk13 transcriptional response (Figure 1b).

The difference between the $p f k 13$ gene expression groups could also be explained by the different ring developmental stage at the 
sample collection time, as recently described by Gibbons et al..$^{20}$ These authors explored the transcriptional profiling of a recombinant $K 13$ strain, with increased susceptibility to artemisinins, revealing down-regulation of pfk13 expression at early rings and upregulation at the early trophozoite stage. They could distinguish this short period of stage development through a full transcriptomic approach and not by microscopic observation of morphological changes, constraining the validation of this hypothesis in our study.

A Kaplan-Meier survival analysis using the $\mathrm{PC}_{50 \%}$ for the two groups ( $p f k 13<1$ and $p f k 13>1$ ) revealed a significant difference in survival time ( $\mathrm{PC}_{50 \%}$ medians: $p \mathrm{fk} 13<1=7.1 \mathrm{~h}$; pfk13>1=1.9 h) (Figure 1c), demonstrating that there is a prognostic value of the K13 transcriptional profile to predict the PC time.

A larger study with full transcriptomic approach would be needed to dissect this phenotypic clearance outcome more comprehensively.

Our study thus revealed the $p f k 13$ transcripts as a potential additional factor relevant for in vivo PC outcome of ACT, at least in the scenario of artemether-lumefantrine managing PfK13 WT African parasites.

\section{Acknowledgements}

The authors thank all the children and their parents for their participation in this study.

\section{Funding}

This work was supported by the Swedish Research Council (VR-20143134); the Swedish International Development Cooperation Agency (SWE-200-165); the Northern Portugal Regional Operational Programme (NORTE 2020), under the Portugal 2020 Partnership Agreement, through the European Regional Development Fund (NORTE-01-0145-FEDER000013); and the Fundação para a Ciência e Tecnologia (FCT) (SFRH/BD/ $129769 / 2017$ to M. S., IF/00143/2015 to P. E. F., PD/BD/127826/2016 to C. C. and SFRH/BPD/76614/2011 to M. I. V.).

\section{Transparency declarations}

None to declare.

\section{References}

1 Dondorp AM, Nosten F, Yi P et al. Artemisinin resistance in Plasmodium falciparum malaria. N Engl J Med 2009; 361: 455-67.

2 Noedl H, Se Y, Schaecher K et al. Evidence of artemisinin-resistant malaria in western Cambodia. N Engl J Med 2008; 359: 2619-20.

3 Amato R, Lim P, Miotto $\mathrm{O}$ et al. Genetic markers associated with dihydroartemisinin-piperaquine failure in Plasmodium falciparum malaria in Cambodia: a genotype-phenotype association study. Lancet Infect Dis 2017; 17: 164-73.
4 Witkowski B, Duru V, Khim N et al. A surrogate marker of piperaquineresistant Plasmodium falciparum malaria: a phenotype-genotype association study. Lancet Infect Dis 2017; 17: 174-83.

5 United Nations Sustainable Development Knowledge Platform. Transforming Our World: The 2030 Agenda for Sustainable Development. https://sustainabledevelopment.un.org/post2015/transformingourworld.

6 Ariey F, Witkowski B, Amaratunga C et al. A molecular marker of artemisinin-resistant Plasmodium falciparum malaria. Nature 2014; 505: 50-5.

7 Straimer J, Gnadig NF, Witkowski B et al. Drug resistance. K13-propeller mutations confer artemisinin resistance in Plasmodium falciparum clinical isolates. Science 2015; 347: 428-31.

8 Mukherjee A, Bopp S, Magistrado P et al. Artemisinin resistance without pfkelch13 mutations in Plasmodium falciparum isolates from Cambodia. Malar J 2017; 16: 195.

9 Carlsson AM, Ngasala BE, Dahlstrom S et al. Plasmodium falciparum population dynamics during the early phase of anti-malarial drug treatment in Tanzanian children with acute uncomplicated malaria. Malar J 2011; 10: 380.

10 Veiga MI, Ferreira PE, Schmidt BA et al. Antimalarial exposure delays Plasmodium falciparum intra-erythrocytic cycle and drives drug transporter genes expression. PLoS One 2010; 5: e12408.

11 Ngwa CJ, Kiesow MJ, Papst O et al. Transcriptional profiling defines histone acetylation as a regulator of gene expression during human-tomosquito transmission of the malaria parasite Plasmodium falciparum. Front Cell Infect Microbiol 2017; 7: 320.

12 Bozdech Z, Llinas M, Pulliam BL et al. The transcriptome of the intraerythrocytic developmental cycle of Plasmodium falciparum. PLoS Biol 2003; 1: E5.

13 Magallon-Tejada A, Machevo S, Cistero P et al. Cytoadhesion to gC1qR through Plasmodium falciparum erythrocyte membrane protein 1 in severe malaria. PLoS Pathog 2016; 12: e1006011.

14 Livak KJ, Schmittgen TD. Analysis of relative gene expression data using real-time quantitative PCR and the 2(-Delta Delta $C(T)$ ) Method. Methods 2001; 25: 402-8.

15 Miotto O, Amato R, Ashley EA et al. Genetic architecture of artemisininresistant Plasmodium falciparum. Nat Genet 2015; 47: 226-34.

16 Kamau E, Campino S, Amenga-Etego L et al. K13-propeller polymorphisms in Plasmodium falciparum parasites from sub-Saharan Africa. J Infect Dis 2015; 211: 1352-5.

17 Ouattara A, Kone A, Adams M et al. Polymorphisms in the K13-propeller gene in artemisinin-susceptible Plasmodium falciparum parasites from Bougoula-Hameau and Bandiagara, Mali. Am J Trop Med Hyg 2015; 92: 1202-6.

18 Mok S, Ashley EA, Ferreira PE et al. Drug resistance. Population transcriptomics of human malaria parasites reveals the mechanism of artemisinin resistance. Science 2015; 347: 431-5.

19 Mbengue A, Bhattacharjee S, Pandharkar T et al. A molecular mechanism of artemisinin resistance in Plasmodium falciparum malaria. Nature 2015; 520: 683-7.

20 Gibbons J, Button-Simons KA, Adapa SR et al. Altered expression of K13 disrupts DNA replication and repair in Plasmodium falciparum. BMC Genomics 2018; 19: 849. 